DOI: 10.20472/IAC.2017.33.051

\author{
JIYOUNG OH \\ Inje University, Korea, Republic of \\ HYUN JOO KWON \\ Purdue University, United States \\ HEYKYUNG PARK \\ Inje University, Korea, Republic of
}

, United States

\title{
EXPERIMENTAL STUDY ON THE DIFFERENCES OF AUTOMATIC NERVOUS SYSTEM AND EMOTIONAL RESPONSE IN COLOR ENVIRONMENT
}

\begin{abstract}
:
The purpose of this study is to examine the differences between the automatic nervous system and the emotional response according to the tone changes in the color environment through experiment. It also identified color plan establishment conditions considering the differences between user's physiological response and psychological reaction in the environment.

An experimental environment set up with pale, bright and vivid $\mathrm{R}$ and $\mathrm{G}$ color tone that selected from NCS color system. Five males and Five females took the color experiment with the heart rate variability device that measuring their heart bit and autonomic nervous system for determining the human body physiology response. After completing the experiment, the response of psychological from the change of the color environment was investigated by conducting the one-on-one in-depth interview.

The result of the investigation is as follows.

First, the response of the autonomic nervous system showed that there was a significant difference between male and female. Male tend to have a decreasing pulse while changing pale $\mathrm{G}$ color to vivid $\mathrm{G}$ color environment. Bright tone increases the LF index of females. There is an opposite result between the male and female for the LF/HF index in the G color tone environment. The noteworthy difference between male and female was shown in the LF and LF/HF of $R$ color tone environment and $\mathrm{LF} / \mathrm{HF}$ of $\mathrm{G}$ color tone environment.

Second, female increased LF, LF/HF index in the bright R color. It was found that stress such as anxiety and frustration were observed in the bright tone rather than the vivid and pale tone. Also, most female's responded that they had a nervous feeling and frustration in the interview after the experiment. Therefore, it is expected that the use of bright tone color should be considered in spaces that are used mostly by female.

Third, the emotional response to the $\mathrm{R}$ color was consistent with that of the female, whereas the psychological response to the $\mathrm{G}$ color did not match with that of both the male and female. The psychological response was different according to the individual experience, preference, and taste. In particular, there were cases that the emotional response to vivid $\mathrm{G}$ color appears to be opposite, so it is expected that users' preference will be greatly changed when vivid tone color is used.
\end{abstract}




\section{Keywords:}

Automatic nervous system response, Emotional response, Color environment

JEL Classification: Q51 